\title{
REVIEW
}

\section{Sepsis guideline implementation: benefits, pitfalls and possible solutions}

\author{
Niranjan Kissoon \\ This article is one of ten reviews selected from the Annual Update in Intensive Care and Emergency Medicine 2014 and co-published as a series \\ in Critical Care. Other articles in the series can be found online at http://ccforum.com/series/annualupdate2014. Further information about the \\ Annual Update in Intensive Care and Emergency Medicine is available from http://www.springer.com/series/8901.
}

\section{Introduction}

Clinical practice guidelines are useful in improving quality of care and outcomes, reducing inappropriate variation in practice, promoting efficient use of resources, informing and empowering patients and informing public policy. However, difficulties arise when guidelines are poorly introduced into routine daily practice and, as a consequence, many patients do not receive the care intended or receive harmful or unnecessary care [1].

Many guidelines have been formulated for the treatment of sepsis in children and adults [2]-[6]. These guidelines emphasize early recognition and aggressive treatment of the patient with sepsis in order to improve outcomes. However, the context in which a guideline is to be used is important and to a large extent determines whether it will be implemented successfully [2], [3], [6][8]. Thus, in an attempt to make sepsis guidelines relevant in both resource-poor and resource-rich environments, the level of resources in various settings have been taken into account and guidelines have been formulated to suit both resource rich and poor regions of the world [2]-[4]. Sepsis guidelines for children have also been designed to accommodate both resource and skill sets for countries with varying under-five mortality rates (Figure 1 ) and to accommodate resources for monitoring and treatment from district clinics to tertiary care facilities [2] (Figure 2), while guidelines for sepsis management of both adults and children have been proposed by the Global Intensive Care Working Group of the European Society of Intensive Care Medicine [3]. In addition, tremendous effort has been expended in revising the Surviving Sepsis Campaign guidelines to include new evidence since its previous iteration in 2008 [4]. Although these efforts are laudable, adherence to these guidelines has met with mixed results in both resource poor and

*Correspondence: nkissoon@cw.bc.ca

British Columbia Childrens Hospital, BC V6H 3V4 Vancouver, Canada rich regions. Therefore, while resources to implement guidelines are important, other factors beyond resources may also mitigate against successful adoption. This manuscript will address some of these issues. It will outline the benefits of compliance with sepsis guidelines, the published experience with compliance, possible reasons for poor compliance and offer some possible solutions to improve compliance and ultimately patient outcomes.

\section{Benefits of compliance with sepsis guidelines}

There is no doubt that adherence with guidelines is associated with better outcomes. Indeed, adherence to the American College of Critical Care Medicine (ACCM) guidelines for children has led to a $30 \%$ decrease in mortality when the guidelines for initial resuscitation were followed by physicians in community hospitals [9]. Moreover, a decrease of $27 \%$ in mortality was seen in children managed according to ACCM guidelines, including central venous oxygen saturation $\left(\mathrm{ScvO}_{2}\right)$ directed therapy, in an intensive care unit [10]. Adherence to sepsis guidelines in a pediatric emergency department in Texas resulted in a decrease in the need for mechanical ventilation and vasoactive agents and a decrease in mortality from 4 to $2.5 \%$ [11]. Guideline adherence in children with sepsis resulted in a $57 \%$ reduction in pediatric intensive care unit (PICU) hospital length of stay in Boston [12], while in Utah increasing compliance with sepsis guidelines resulted in a decrease in mortality from 8.4 to $3.5 \%$ [13]. In all these instances, although outcomes improved with compliance, adherence to some elements of the guideline was less than optimal and in many instances the entire bundle was provided to few patients. Similar findings have been seen in adults in the Surviving Sepsis Campaign in which there was a significant decrease in mortality with adherence to resuscitation and management bundles [14]. Unadjusted hospital mortality decreased from $37 \%$ to $30.8 \%$ over 


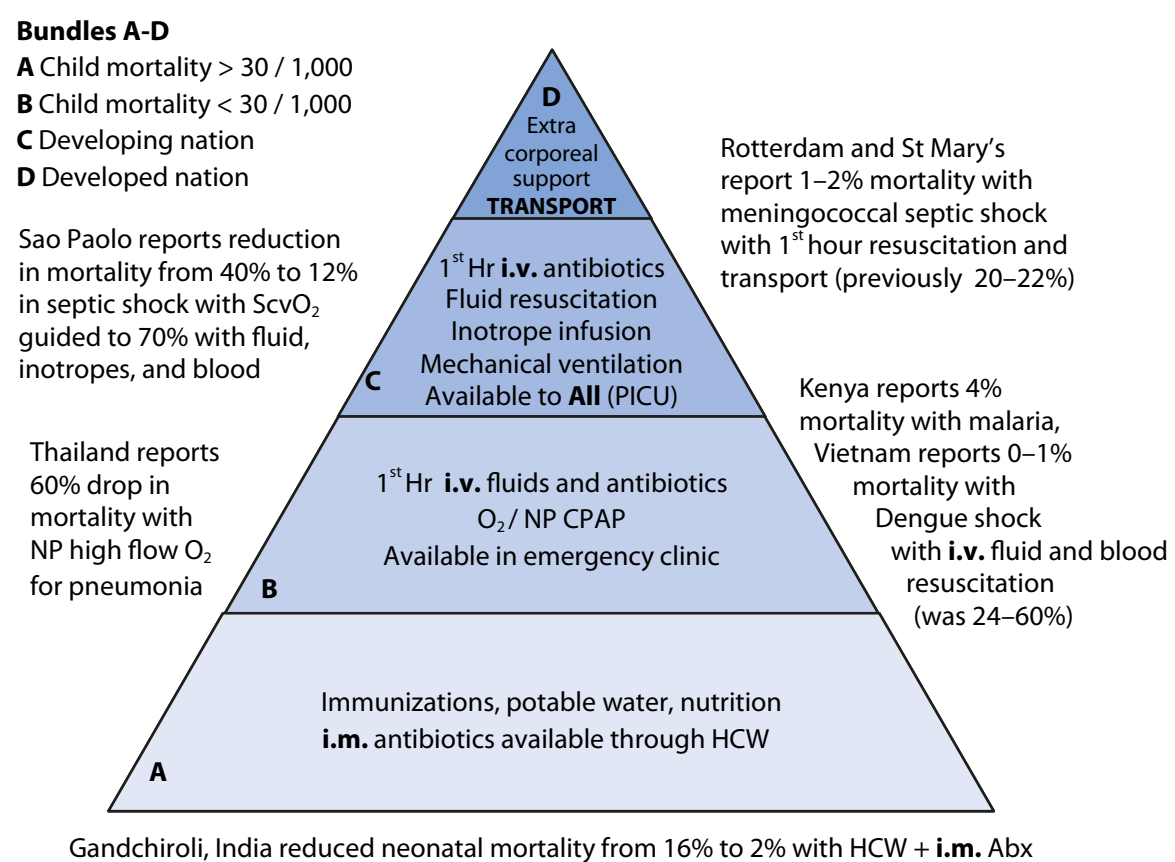

Figure 1. Global Newborn and Child Sepsis guidelines: Proposed Bundles A-D align with local resources. CPAP: continuous positive airway pressure; i.v: intravenous; i.m.: intramuscular; $\mathrm{ScVO}_{2}$ : central venous oxygen saturation; Abx: antibiotics; PRBCs: packed red blood cells; PICU: pediatric intensive care unit; $\mathrm{HCW}$ : healthcare worker.

Continuous cardiorespiratory monitoring \& advanced laboratory support, CVP line, supplies of inotropes/ vasoactive agents, skil to use inodilators, an intensivist

Supplies of ET tubes, ventilation bags, skill for intubation \& manual ventilation, pulse oximeter non-invasive monitor, a pediatrician

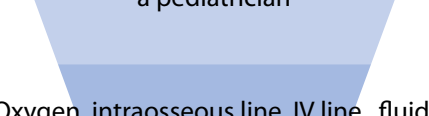

Oxygen, intraosseous line, IV line, fluid supplies, urinary catheter, first line i.v. antibiotics, ET tubes, dopamine*,

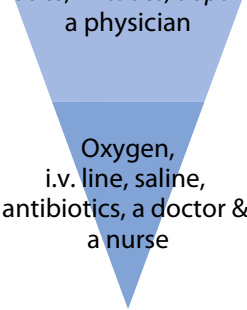

Resources

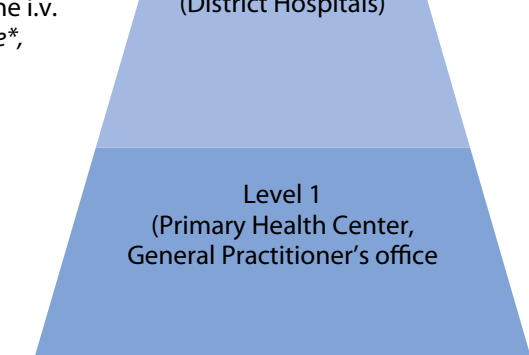

Level of care

CVP line
Manual/mechanical
ventilation
Optimization of fluids,
vasoactive therapy and
oxygen delivery
Cultures \& source
control (surgery)
Dopamine, dobutamine
Intubation
Manual ventilation
Nasal prong cPAP
Rapid fluid infusion
Intubation
Dopamine i.v.
Correction of
hypoglycemia

O, by free flow/face
mask/nasal prongs
i.v. access
Fluid infusion

CVP monitoring Continuous monitoring of MAP, perfusion pressure, invasive $\mathrm{BP}$ $\mathrm{ScvO}_{2}$ measurements Blood gases \& lactate

Continuous EKG Pulse oximetry

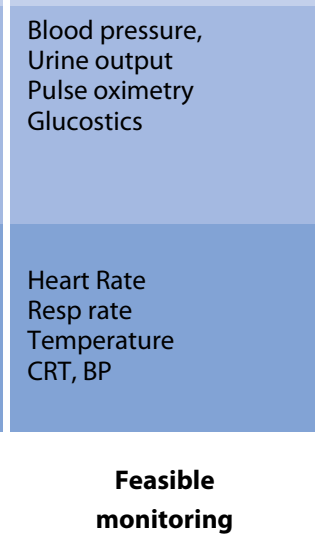

Figure 2. Resources and interventions expected to be made available at different levels of health care facilities in resource-poor economies. CVP: central venous pressure; MAP: mean arterial pressure; $\mathrm{ScvO}_{2}$ : central venous oxygen saturation; BP: blood pressure; ET: endotracheal. * Not available universally at all level 2 facilities. 
2 years $(\mathrm{p}=0.001)$, offering optimism for further improvement in outcomes [14]. The experience of the World Federation of Pediatric Intensive and Critical Care Societies (WFPICCS) initiative in children is similar with significant decreases in mortality with compliance with the resuscitation bundle (OR 0.40, 95 \% CI 0.19-0.72 $\mathrm{p}<0.004$ ) and compliance with the management bundle was also associated with a decrease in mortality (OR 0.30 , $95 \%$ CI 0.10-0.80, p < 0.018) [2]. These benefits applied to children in both the developed and the developing world. In addition, although outcomes improved as resources increased, adherence did not differ markedly suggesting that resources, while important, are not the only determinant of compliance.

\section{Success with adherence to sepsis guidelines}

In the WFPICCS endeavor, resuscitation bundle compliance ranged from 24-52 \% while management bundle compliance range from 10-25\% across centers [2]. Similarly, in the Surviving Sepsis Campaign, compliance with the entire management bundle started at $18 \%$ and increased to approximately $36 \%$ at the end of two years [14]. In children in areas that were adequately resourced, the news is no better. Indeed, there was $19 \%$ adherence to the resuscitation bundle at Boston Children's Hospital with significant delays in intravenous fluid administration and inotrope administration [13]. In Utah and Texas, while intense efforts achieved an increase in compliance, this was still suboptimal, with the highest compliance $80 \%$ - for intravenous fluids, antibiotic administration and lactate evaluation [11], [12]. Delayed recognition and delayed intravenous fluids and inotropes were also reported, along with a $36 \%$ adherence to pre-PICU care, in a follow-up assessment of treatment guidelines for meningococcemia in the UK [15]. In India, a survey reported $12 \%$ adherence to the ACCM guidelines among physicians; this low adherence was attributed mostly to lack of skills and knowledge [16]. Adherence to guidelines has also been poor in other parts of the world, including in Africa, where less than $50 \%$ of the Surviving Sepsis Campaign guidelines were implemented; the predominant reasons were resource-limitations and lack of education [7]. In Asia (China, Hong Kong, India, Malaysia, Singapore and South Korea) adherence to Surviving Sepsis Campaign guidelines ranged from 5-15 \% [17]. Low adherence to sepsis guidelines was also found in Germany, where there was a perception reality gap; physicians perceived that adherence to low tidal volume ventilation was $80 \%$, whereas in reality it was $2.6-17 \%$. Similarly, the perception of adherence to glycemic control was $66 \%$ whereas the reality was $6 \%$ [18]. Suboptimal management related to lack of adherence to sepsis guidelines has also been reported in children in France, England, and Australia [19]-[21]. In most cases, suboptimal management resulted from underestimation of disease severity, physician delay in administrating antibiotics or fluids, insufficient fluid administration and inadequate inotropic support.

\section{Reasons for poor adherence}

Major contributors to poor adherence to guidelines are many fold and include failure to recognize sepsis, lack of familiarity or lack of awareness of the sepsis guideline, lack of agreement with the specific guideline, or lack of agreement with guidelines in general, as well as lack of motivation [22], [23]. In addition there are many external barriers to guideline implementation. For instance, the characteristics of the guidelines may render them impractical to implement - in some cases they are too detailed and try to address all eventualities, whereas in others they may suggest resources, such as laboratory tests, methods of monitoring and treatment options, that are not available locally [3], [6], [7]. Environmental factors, such as lack of time, lack of resources, lack of reimbursement and organizational constraints, may also preclude adoption of guidelines. For instance, in areas where there are critical staff shortages, it is unreasonable to place further burdens, such as frequent monitoring and documentation, which are the standard of care in areas with substantially more resources. In many areas of the world, white blood cell counts to determine systemic inflammatory response syndrome (SIRS) criteria, laboratory capabilities for blood culture and pulse oximetry or supplemental oxygen are not readily available [6], [7].

Poor guideline adoption may also be due to the fact that incentives may not be aligned to the behavior. There are also concerns which lead to skepticism that guidelines may be subject to biases (used as a financial and marketing tool). Doubts about the evidence on which a guideline is based stems from skepticism of the composition of the panels of experts that mold these judgments. While guideline users could sometimes adjust for these biases, in some cases the values and goals and conflicts are not explicit to allow for any adjustments. Moreover, some have argued that there are too many sepsis guidelines and some are out of date and present conflicting information. A major concern in the United States is the fact that these guidelines may be turned into performance measures to critique the quality of physician care and even dictate hospital accreditation.

In our local experience, clinicians were skeptical when a sepsis guideline was introduced because they felt that screening for sepsis in the emergency department was not necessary because their triage system was robust enough to detect sepsis. Others felt their pediatric early warning systems served the same purpose on the wards, and still others felt that introduction of the sepsis guideline implied that they were managing sepsis 


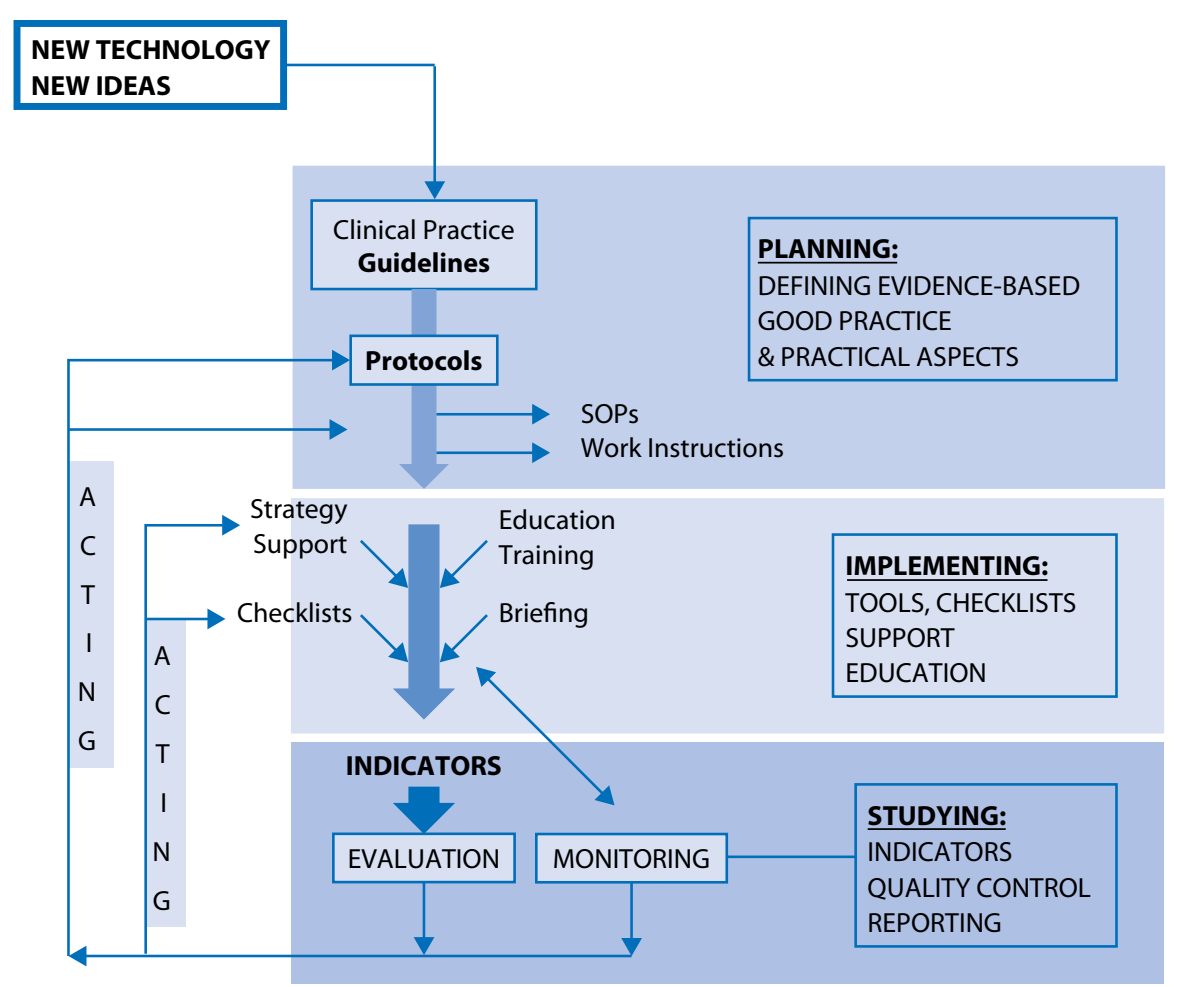

Figure 3. Clinical practice guidelines. Preparation, implementation, evaluation and revision are all important for successful adoption.

incorrectly beforehand. These reasons for skepticism are not unique to any single institution or any particular guideline and imply that crafting a resource-appropriate guideline is an important process but without attention to cultural issues, implementation and adoption are likely to be less than optimal (Figure 3 [24]). Another area that has hindered adoption and sustainability is the failure to measure meaningful outcomes and share the information widely with team members.

\section{A framework for crafting a sepsis guideline}

In order to circumvent some of the barriers outlined above, as a first step, the guideline writing process should be rigorous and transparent. It is important that appropriate clinicians and policy makers be involved early in the discussion pertaining either to crafting a guideline de novo or to adapting an existing guideline, such as the WFPICCS society or Surviving Sepsis Campaign guidelines. This is important because failure to invite the appropriate broad representation to the table will likely lead to frustration, suspicion and ultimately failure. For example, in our institution an oversight on our part was failure to involve pharmacy representatives at the start of the process even though they are involved in stocking unit doses of antibiotics in the emergency room and ICUs.

The AGREE tool is an example of a tool that provides a roadmap to either create or evaluate a guideline (http:// www.agreetrust.org). Strict adherence to the elements included in such a roadmap will enable all stages to be conducted without missing any important steps, will insure the appropriate team members are involved, and will insure transparency and literature review. It will also allow evaluation of the necessary resources and outcome measures as well as opportunities for revising the guideline. The AGREE tool consists of 6 domains (Table 1) that address all aspects of implementation. Each of these domains controls a series of items (total 23) that guide every step of guideline development and address factors that may preclude adoption. AGREE is not the only tool that serves to assist in guideline development but it is validated, easy to use, widely accepted and comes with an easily accessible training manual.

Barriers to successful guideline implementation are summarized in Figure 4. Poor adherence can be due to inherent flaws in the process used in preparing the guidelines as outlined above, but just as important are the strategies used in implementation. Moreover, if quality control indicators for evaluation and monitoring are not appropriate and agreed on, monitoring will be haphazard and inadequate and provide meaningless information. This poses a problem in that if outcome measures are not monitored diligently, it is very difficult to determine the effectiveness of the guideline and to act to revise the guideline and protocol or address 
Table 1 The Appraisal of Guidelines for Research and Evaluation (AGREE)

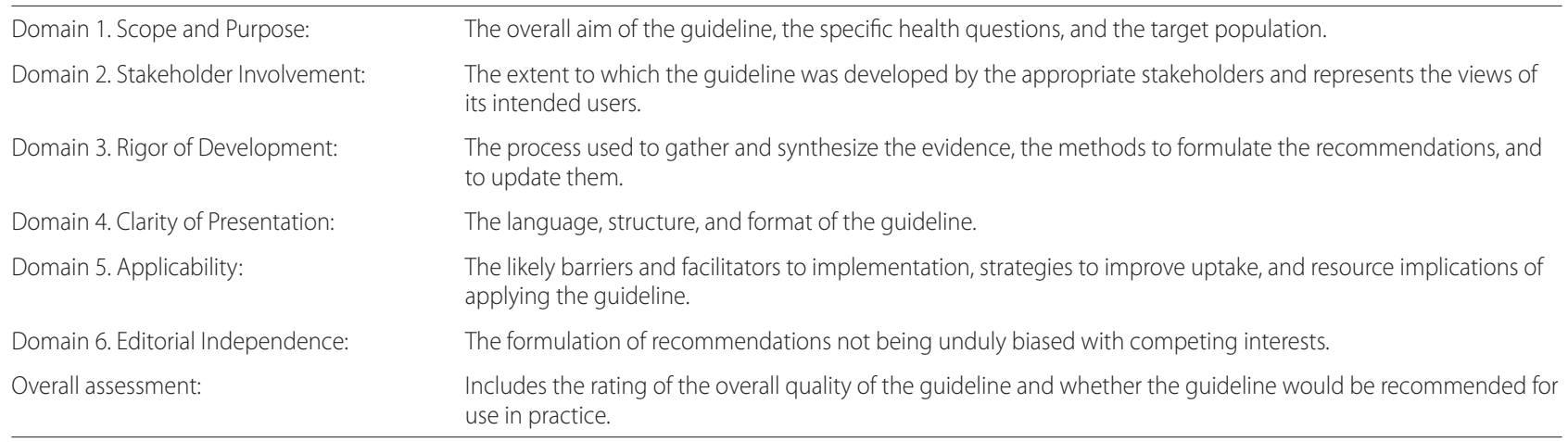

deficiencies in guideline implementation. Feedback loops using rapid PDSA (Plan, Do, Study, Act) cycles are important to continuously improve the guideline itself as well as to address cultural, resource and care process issues. In addition, with the advent of new technology, the framework used should include processes to incorporate these new facets to improve the care processes (Figure 3).

\section{Obstacles and solutions in implementation}

In many parts of the developing world, poor guideline adherence is due to a lack of resources such that those who are responsible for implementation of the guideline are unable to do so. The obstacles in sepsis guideline implementation are unique to the local environment and hence an environmental scan is important to highlight the deficiencies that need to be addressed [7]. In some areas, the deficiencies are obvious and mostly relate to a lack of personnel and supplies. For example, in many areas of the world, human resources and equipment and supplies, such as antimicrobials, fluids and oxygen, are lacking or sporadically available. Essential staff, equipment and supplies, therefore, need to be provided for successful implementation [25], [26]. Deficiencies such as these should be brought to the attention of clinicians in positions of authority and policymakers so that they can be addressed. There are limits to the resources that can be invested and this also highlights the importance of crafting guidelines that are realistic to the local context. For example, expectations regarding laboratory monitoring for diagnosis and response to therapy are context dependent; in many areas of the world, blood counts are rarely available and monitoring may involve vital signs and pulse oximetry only.

Overcoming some of the major challenges also requires creativity especially when resources are limited. For example, lack of time and staff is a major barrier that can be somewhat circumvented by creating standard operating procedures [5], [9], [10]-[13], [27]. Sepsis screening, for example, should be incorporated into the triage process in emergency departments rather than be done separately. In as much as possible, sepsis screening and treatment must be standard work and hence also be congruent with early warning scores and systems [28]. In addition, creating sepsis carts and standard flow sheets can also assist in standardizing and avoiding duplication of work. Prepacked kits consisting of intravenous cannulas and fluid administration sets as well as readily accessible essential drugs and fluids may also encourage greater compliance. Specialized training and equipment is also an issue but in resource limited environments less invasive monitoring, use of peripheral inotropes and procedural training may be needed.

Lack of education, including recognition of signs and symptoms of sepsis, is an issue that may lend to poor compliance and needs to be addressed. With little training, even patients and families, village health workers and non-physician clinicians, such as anesthetic assistants and nurses, can be taught to recognize and treat sepsis, as reported from Malawi [25], [26]. Familiarity with the guideline should be insured as well as insuring that several versions are not in circulation. Courses that are sanctioned by the World Health Organization, such as the Emergency Triage Assessment and Treatment and the Integrated Management of Adolescent and Adult Illness, are useful in resource poor areas and address critical illness as well as sepsis [6], [29].

What poses greater difficulty is attitudinal and cultural aversion to guideline adoption and adherence (Figure 2). To overcome this barrier, advocacy, leadership and support is necessary from clinicians and policy makers alike to address all facets of process and structure (Figure 5). We have found that involvement of an anthropologist to assist in identifying causes of aversion and to facilitate change can enable dramatic positive gains. Attempts at building a community of practice emphasizing shared values and goals and shared learning experiences can also be a useful robust enabler. A community of practice may generate innovative ideas to circumvent resource limitations, ensure staff engagement and advance educational efforts [30], [31]. A community of practice can foster collaboration among medical specialties, such as the 


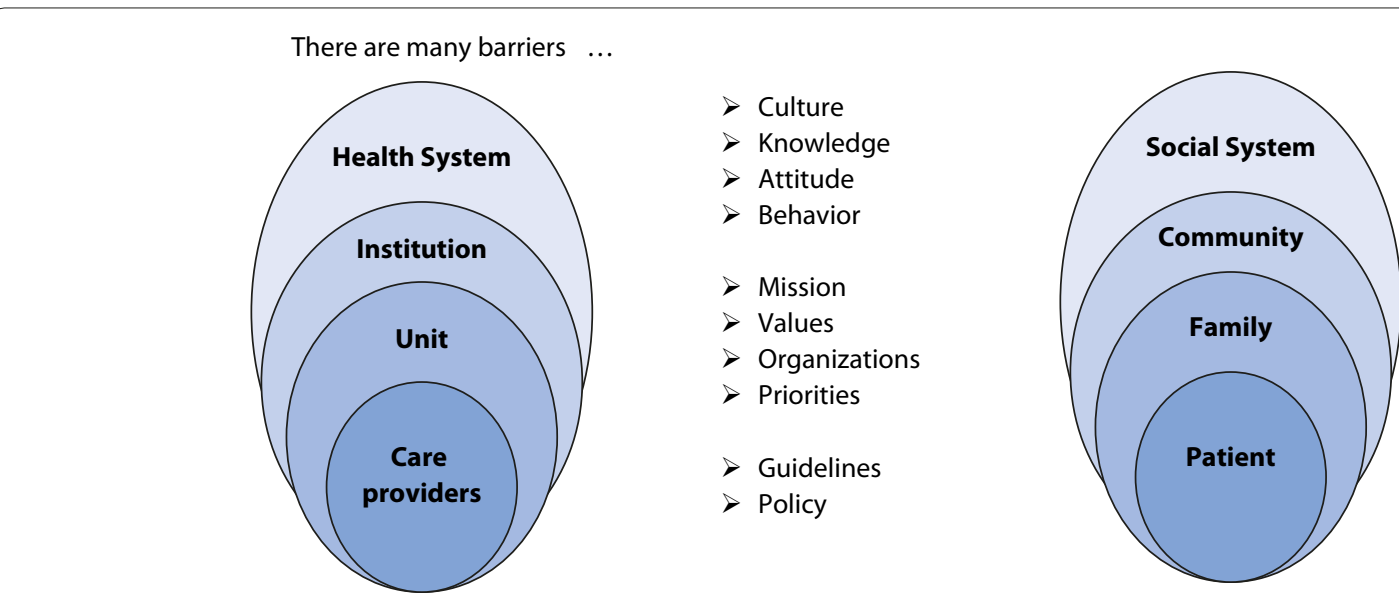

Figure 4. Barriers to successful guideline implementation. All must be addressed for successful adoption of guidelines.

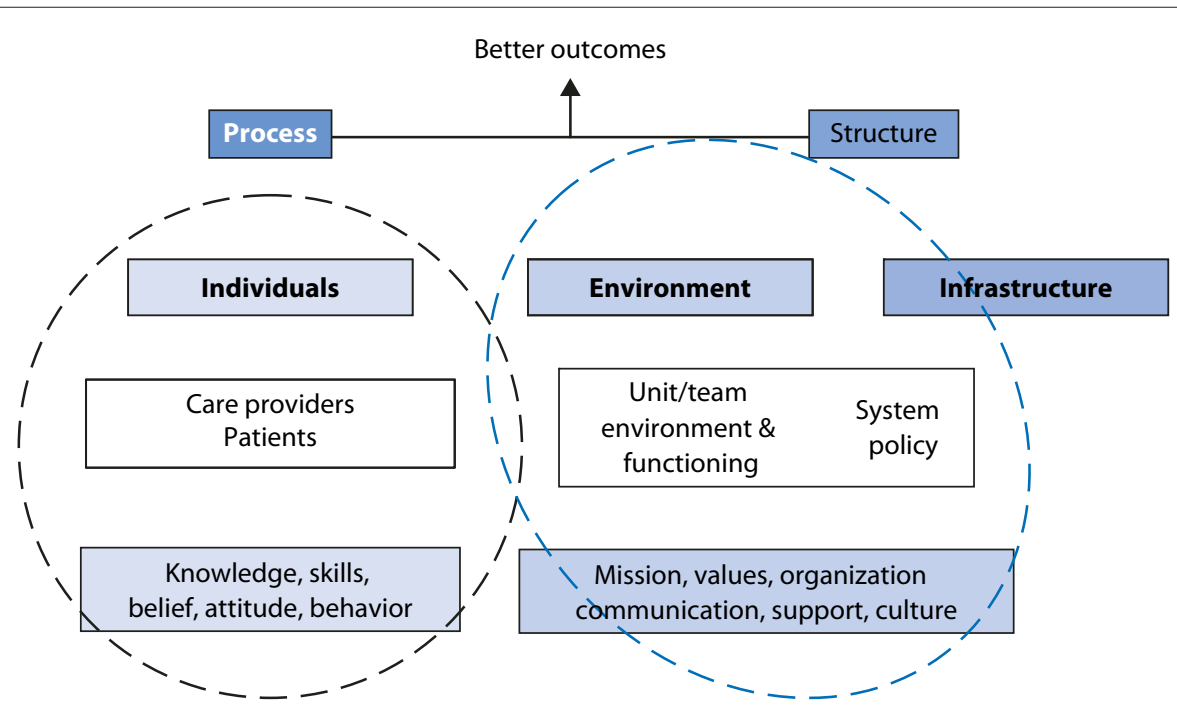

Figure 5. Improving performance in sepsis management requires attention to both process and structure. Individuals, the environment and infrastructure must be conducive to successful guideline adoption.

emergency department and the ICU, through standard operating practices. A team model is associated with an increase in compliance with guideline of $80 \%$ versus $40 \%$ in the non-team model [8]. The community can also foster early referral to the ICU and involvement of sepsis crash teams and rapid response teams in the care of patients with sepsis. A stewardship program can enable a robust quality assurance program and the designation of an ambassador and feedback systems can facilitate sustainability [30], [32]. Ultimately, ensuring guideline adherence requires a gargantuan effort from those in leadership such that the culture of the organization changes to embrace guidelines as part of standard work. Moreover, commitment has to be ongoing because a decline in vigilance is likely to result in loss of previous gains [5]. The rewards for sustained dogged effort can be improved guideline adherence both in resource-rich and resource-poor environments.

\section{Conclusion}

Guidelines are useful in improving the quality of care and outcomes, reducing inappropriate variation in practice and promoting efficient use of resources. However, the benefits are hampered by poor adoption in both resource-rich and resource-poor environments. Adoption and adherence to guidelines is hampered by many factors, including the very nature of the process used in preparing the guidelines, as well as clinicians' skepticism, cultural aversion to guidelines and resource limitations that preclude implementation.

In order to circumvent these issues it is suggested that a uniform and transparent inclusive process be used to 
craft the guidelines. The AGREE tool is one example of a system to insure that guideline development is rigorous. Strict adherence to its elements will enable all steps of the guideline process to be conducted, will involve the appropriate team members, and will insure transparency and literature review. Such tools also include systems to allow evaluation of resources needed and outcome measures as well as opportunities for revising the guideline. The guidelines should also be crafted with a knowledge of the context they would be employed. An environmental scan to identify the possible barriers to implementation in any setting is important. The most common barriers are lack of personnel and resources for carrying out the steps required for guideline adherence. These barriers should be addressed early in the implementation stage for guideline adoption to be successful. Rigorous attention should be paid to outcome measures to determine adherence to guidelines as well as relevance to patient care. Many of the barriers to guideline adherence can be overcome by close adherence to the culture of the environment in which the guideline will be adopted. Developing a community of practice in which all clinicians are involved in the development and promotion of the guidelines may ensure their success. Ensuring that a guideline is successfully adopted requires a tremendous investment of resources and effort. However, the favorable outcomes associated with guideline adherence far outweigh the effort that is needed for successful implementation.

\section{List of abbreviations used}

Abx: antibiotics; ACCM: American College of Critical Care Medicine; BP: blood pressure; ET: endotracheal; $\mathrm{Cl}$ : confidence interval; CPAP: continuous positive airway pressure; CVP: central venous pressure; HCW: healthcare worker; i.m.: intramuscular; i.v.: intravenous; MAP: mean arterial pressure; OR: odds ratio; PICU: pediatric intensive care unit; pRBCs: packed red blood cells; $\mathrm{SCVO}_{2}$ : central venous oxygen saturation; $\mathrm{SCvO}_{2}$ : central venous oxygen saturation; SIRS: severe inflammatory response syndrome; WFPICCS: World Federation of Pediatric Intensive and Critical Care Societies.

\section{Competing interests}

The author declares that they have no competing interests.

\section{Declarations}

Publication of this article was funded by BC Children's Hospital and the University of British Columbia Professor in Acute and Critical Care - Global Child Health.

\section{Published: 18 March 2014}

\section{References}

1. Grol R, Grimshaw J: From best evidence to best practice: effective implementation of change in patients' care. Lancet 2003, 36:1225-1230.

2. Kissoon N, Carcillo JA, Espinosa V, Argent A, Devictor D, Madden M, Singhi S, van der Voort E, Latour J, Global Sepsis Initiative Vanguard Center Contributors: World Federation of Pediatric and Intensive Care Societies: Global Sepsis Initiative. Pediatr Crit Care Med 2011, 12:494-503.

3. Dunser MW, Festic E, Dondorp A, Kisson N, Ganbat T, Kwizera A, Haniffa R, Baker T, Schultz MJ, Global Intensive Care Working Froup of European Society of Intensive Care Medicine: Recommendations for sepsis management in resource-limited settings. Intensive Care Med 2012, 38:557-574.

4. Dellinger RP, Levy MM, Rhodes A, Anane D, Gerlach H, Opal SM, Sevransky JE,
Sprung CL, Douglas IS, Jaeschke R, Osborn TM, Nunnally ME, Townsend SR, Reinhart K, Kleipell RM, Angus DC < Deutchman CS, Machado DR, Rubenfeld GD, Webb S, Beale RJ, Vincent JL, Moreno R, Surviving Sepsis Campaign Guidelines Committee including The Pediatric Subgroup: Surviving Sepsis Campaign: international guidelines for management of severe sepsis and septic shock 2012. Intensive Care Med 2013, 39:165-228.

5. Plambech MZ, Lurie Al, Ipsen HL: Initial successful implementation of sepsis guidelines in an emergency department. Dan Med J 2012, 59:A4545.

6. Jacob ST, Lim M, Banura P, Bhagwanjee S, Bion J, Cheng AC, Cohen H, Farrar J, Gove S, Hopewell P, Moore CC, Roth C, West TE: Integrating sepsis management recommendations into clinical care guidelines for district hospitals in resource-limited settings: the necessity to augment new guidelines with future research. BMC Med 2013, 11:107.

7. Baelani I, Jochberger S, Laimer T, Rex C, Baker T, Wilson IH, Grander W, Dünser $M W$ : Identifying resource needs for sepsis care and guideline implementation in the Democratic Republic of the Congo: a cluster survey of 66 hospitals in four eastern provinces. Middle East J Anesthesiol 2012. 21:559-575.

8. Na S, Kuan WS, Mahadevan M, Li CH, Shrikhande P, Ray S, Batech M, Nguyen $H B$, ATLAS Investigators: Implementation of early goal-directed therapy and the surviving sepsis campaign resuscitation bundle in Asia. Int I Qual Health Care 2012, 24(5):452-462.

9. Han YY, Carcillo JA, Dragotta MA, Bills DM, Watson RS, Westerman ME, Orr RA: Early reversal of pediatric-neonatal septic shock by community physicians is associated with improved outcome. Pediatrics 2003, 112:793-799.

10. De Oliveira CF, deOliveira DS, Gottschald AF, Moura JD, Costa GA, Ventura AC, Fernandes JC, Vaz FA, Carcillo JA, Rivers EP, Troster EJ: ACCM/PALS hemodynamic support guidelines for paediatric septic shock: an outcomes comparison with and without monitoring central venous oxygen saturation. Intensive Care Med 2008, 34:1065-1075.

11. Cruz AT, Perry AM, Williams EA, Graf JM, Wuestner ER, Patel B: Implementation of goal-directed therapy for children with suspected sepsis in the emergency department. Pediatrics 2011 127:e758-e766.

12. Larsen GY, Mecham N, Greenbert R: An emergency department septic shock protocol and care guideline for children initiated at triage. Pediatrics 2011, 127:e1585-e1592.

13. Paul R, Neuman MI, Monuteaux MC, Melendez E: Adherence to PALS sepsis guidelines and hospital length of stay. Pediatrics 2012, 130:e273-e280.

14. Levy MM, Dellinger RP, Townsend SR, Linde-Zwirble WT, Marshall JC, Bion J, Schorr C, Artigas A, Ramsay G, Beale R, Parker MM, Gerlach H, Reinhart K, Silva E, Harvey M, Regan S, Angus DC, Surviving Sepsis Campaign:The Surviving Sepsis Campaign: results of an international guideline-based performance improvement program targeting severe sepsis. Crit Care Med 2010, 38:367-374

15. Ninis N, Phillips C, Bailey L, Pollock JI, Nadel S, Britto J, Maconochie I, Winrow $A$, Coen PG, Booy R, Levin M: The role of healthcare delivery in the outcomes of meningococcal disease in children: case-control study of fatal and non-fatal cases. BMJ 2005, 330:1475.

16. Santhanam I, Kissoon N, Kamath SR, Ranjit S, Ramesh J, Shankar J: GAP between knowledge and skills for the implementation of the ACCM/PALS septic shock guidelines in India: is the bridge too far? Indian J Crit Care Med 2009, 13:54-58.

17. Phua J, Koh Y, Du B, Tang YQ, Divatia JV, Tan CC, Gomersall CD, Farug MO, Shrestha BR, Gia Binh N, Arabi YM, Salahuddin N, Wahyuprajitno B, Tu ML, Wahab AY, Hameed AA, Nishimura M, Procyshyn M, Chan YH, MOSAICS Study Group: Management of severe sepsis in patients admitted to Asian intensive care units: prospective cohort study. BMJ 2011, 342:d3245.

18. Brunkhorst FM, Engel C, Ragaller M, Welte T, Rossaint R, Gerlach H, Mayer K, John S, Stuber F, Weiler N, Oppert M, Moerer O, Bogatsch H, Reinhart K, Loeffler M, Hartog C, German Sepsis Competence Network (SepNet): Practice and perception - a nationwide survey of therapy habits in sepsis. Crit Care Med 2008, 36(27):199-125.

19. Launay E, Gras-Le Guen C, Martinot A, Assathiany R, Blanchais T, Mourdi N, Aouba A, Bouvier-Colle MH, Rozé JC, Chalumeau M: Suboptimal care in the initial management of children who died from severe bacterial infection: a population-based confidential inquiry. Pediatr Crit Care Med 2010, 11:469-474.

20. Inwalds DP, Tasker RC, Peters MJ, Nadel S: Emergency management of children with severe sepsis in the United Kingdom: the results of the Paediatric Intensive Care Society sepsis audit. Arch Dis Child 2009, 94:348-353. 
21. Mclntyre PB, Macintyre CR, Gilmour R, Wang H: A population based study of the impact of corticosteroid therapy and delayed diagnosis on the outcome of childhood pneumococcal meningitis. Arch Dis Child 2005 90:391-396.

22. Cabana MD, Rand CS, Powe NR, Wu AW, Wilson MH, Abboud PA, Rubin HR: Why don't physicians follow clinical practice guidelines: A framework for improvement. JAMA 1999, 282:1458-1465.

23. Shaneyfelt TM, Centor RM: Reassessment of clinical practice guidelines: go gently into that good night. JAMA 2009, 301:868-869.

24. Leone M, Ragonnet B, Alonso S, Allaouchiche B, Constantin JM, Jaber S, Martin C, Fabbro-Peray P, Lefrant JY, AzuRéa Group: Variable compliance with clinical practice guidelines identified in a 1-day audit at 66 French adult intensive care units. Crit Care Med 2012, 40:3189-3195.

25. Pollach G, Namboya F: Preventing intensive care admissions for sepsis in tropical Africa (PICASTA): and extension of the international pediatric global sepsis initiative: and African perspective. Pediatr Crit Care Med 2013, 14:561-570.

26. Kissoon N: Preventing intensive care admissions for sepsis in tropical Africa: PICASTA - food for thought. Pediatr Crit Care Med 2013, 14:644-645.

27. Barochia AV, Cui X, Vitberg D,Suffredini AF, O'Grady NP, Banks SM, Minneci P, Kern SJ, Danner RL, Natanson C, Eichacker PQ: Bundled care for septic shock: an analysis of clinical trials. Crit Care Med 2010, 39:668-678.
28. Nguyen HB, Corbett SW, Steele R, Banta J, Clark RT, Hayes SR, Edwards J, Cho TW, Wittlake WA: Implementation of a bundle of quality indicators for the early management of severe sepsis and septic shock is associated with decreased mortality. Crit Care Med 2007, 35:1105-1112.

29. Irimu GW, Gathara D, Zurovac D, Kihara H, Maina C, Mwangi J, Mbori-Ngacha D, Todd J, Greene A, English M: Performance of health workers in the management of seriously sick children at a Kenyan tertiary hospital: before and after a training intervention. PLoS One 2012, 7:e39964.

30. Schramm GE, Kashyap R, Mullon JJ, Gajic O, Afessa B: Septic shock: a multidisciplinary response team and weekly feedback to clinicians improve the process of care and mortality. Crit Care Med 2011, 39:252-258.

31. Wenger E: Communities of practice and social learning systems. Organization 2000, 7:225-246.

32. Shorr AF, Micek ST, Jackson WL Jr, Kollef MH: Economic implications of an evidence-based sepsis protocol: can we improve outcomes and lower costs? Crit Care Med 2007, 35:1257-1262.

doi:10.1186/cc13774

Cite this article as: Kissoon N: Sepsis guideline implementation: benefits, pitfalls and possible solutions. Critical Care 2014, 18:207. 